\title{
Some results on value distribution and uniqueness for $q$-shift difference polynomials of meromorphic functions
}

\author{
Harina Pandit Waghamore and Vijaylaxmi Subhas Bhoosnurmath \\ Department of Mathematics, Jnanabharathi Campus, Bangalore University, Bengaluru-560 056, India
}

Received: 21 February 2019, Accepted: 14 August 2019

Published online: 29 September 2019.

\begin{abstract}
In the present paper, we investigate the problem on the value distribution and uniqueness for $q$-shift polynomials of transcendental meromorphic(entire) functions of zero order of the form $f^{m}(z) P_{n}(f(q z+\eta)) f^{(k)}(z)$. By introducing the weighted sharing our results take a new form. We obtain analogous results due to the authors H. Y. Xu, K. Liu and T. B. Cao [25], which is the motivation for our work on the above mentioned combination.
\end{abstract}

Keywords: Transcendental meromorphic functions, q-shift difference polynomials, zero order.

\section{Introduction}

A meromorphic function $f$ will always mean meromorphic in the whole complex plane $\mathbb{C}$. We also assume that the reader has familiarity with the standard notations and basic fundamental results of Nevanlinna Theory such as $T(r, f)$, $m(r, f)$ and $N(r, f)$, the first and second main theorems of Nevanlinna, the lemma of logarithmic derivatives ([11], [26], [28]). For a meromorphic function $f$, we denote $S(r, f)$ any quantity satisfying $S(r, f)=o(T(r, f))$ for all $r$ outside a possible exceptional set of the finite logarithmic measure. Also, we denote $\mathbb{S}(f)$ as the family of all meromorphic functions $\alpha$ such that $T(r, \alpha)=S(r, f)=o(T(r, f))$, where $r \rightarrow \infty$ outside a possible exceptional set of the finite logarithmic measure. We also agree that $\mathbb{S}(f)$ will include all constant functions and $\widehat{\mathbb{S}}:=\mathbb{S}(f) \cup\{\infty\}$. Denote $S_{1}(r, f)$ by any quantity satisfying $S_{1}(r, f)=o(T(r, f))$ for all $r$ on a set of logarithmic density 1 .

Let $f(z)$ and $g(z)$ be two non-constant meromorphic functions. For some $a \in \mathbb{C} \cup\{\infty\}$, if the zeros of $f(z)-a$ and $g(z)-a$ coincide in locations and multiplicities, then we say that $f(z)$ and $g(z)$ share the value $a \operatorname{CM}($ counting multiplicities) and if $f(z)-a$ and $g(z)-a$ coincide in locations only then, we say that $f(z)$ and $g(z)$ share the value $a$ IM(ignoring multiplicities). Also, if $a=\infty$, then the zeros of $f(z)-a$ and $g(z)-a$ becomes the poles of $f(z)$ and $g(z)$ respectively.

The $q$-difference analog of the Nevanlinna theory and their applications on the value distribution of $q$-difference polynomials and $q$-shift-difference equations are being studied in recent years. Especially, for a transcendental meromorphic (resp. entire) function $f(z)$ of order zero, Zhang and Korhonen [30] studied the value distribution of q-difference polynomials of $f(z)$.

Recently, there has been an increase in the interest in study of difference analogue's of Nevanlinna Theory (See [3], [5], [8], [10], [13], [14], [17], [18], [21], [23], [24], [29]). Halburd and Korhonen [8] established a difference analogue of the lemma of logarithmic derivative. Later, the authors Barnett, Halburd, Korhonen and Morgan [2] also established 
difference analogue of the lemma of logarithmic derivative on $q$-difference operators.

In the study of $q$-difference of meromorphic functions, Zhang and Korhonen [30] obtained the following result on the value distribution of $q$-difference polynomial of meromorphic functions.

Theorem 1. [30]. Let $f$ be a transcendental meromorphic (resp. entire) function of zero order and $q$ be non-zero complex constant. Then for $n \geq 6$ (resp. $n \geq 2$ ), $f^{n}(z) f(q z)$ assumes every non-zero value $a \in \mathbb{C}$ infinitely often.

The author's Liu and Qi [20] studied the value distribution for a $q$-shift of the meromorphic function and obtained the following result.

Theorem 2. [20]. Let $f$ be a zero-order transcendental meromorphic function, $n \geq 6, q \in \mathbb{C} \backslash\{0\}, \eta \in \mathbb{C}$, and $R(z) a$ rational function. Then the $q$-shift difference polynomial $f^{n}(z) f(q z+\eta)-R(z)$ has infinitely many zeros.

In 2015, the author's H. Y. Xu, K. Liu and T. B. Cao [25] obtained the following results on finding the zeros of $P(f) f(q z+$ $\eta)=a(z)$ and $P(f)[f(q z+\eta)-f(z)]=a(z)$, where $a(z) \in \mathbb{S}(f) \backslash\{0\}$, let $P(z)=a_{n} z^{n}+a_{n-1} z^{n-1}+\ldots+a_{1} z+a_{0}$ be a non-zero polynomial, where $a_{0}, a_{1}, \ldots, a_{n-1}, a_{n}(\neq 0)$ are complex constants, and let $m$ be the number of the distinct zeros of $P(z)$.

Theorem 3. [25]. Let $f$ be a zero-order transcendental meromorphic (resp. entire) function, $q \in \mathbb{C} \backslash\{0\}, \eta \in \mathbb{C}$. Then for $n>m+4$ (resp. $n>m), P(f) f(q z+\eta)=a(z)$ has infinitely many solutions, where $a(z) \in \mathbb{S}(f) \backslash\{0\}, P(f)$ and $m$ are stated as above.

Theorem 4. [25]. Let $f$ be a zero-order transcendental meromorphic (resp. entire) function, $q \in \mathbb{C} \backslash\{0\}, \eta \in \mathbb{C}$. Then for $n>m+6($ resp. $n>m+2), P(f)[f(q z+\eta)-f(z)]=a(z)$ has infinitely many solutions, where $a(z) \in \mathbb{S}(f) \backslash\{0\}, P(f)$ and $m$ are stated as above.

Some recent results for the uniqueness of the difference and the $q$-difference of meromorphic functions were obtained by the authors ([12], [13], [17], [19], [22], [28], [29]).

In 2010, Zhang and Korhonen [30], obtained the following result.

Theorem 5. [30]. Let $f(z)$ and $g(z)$ be two transcendental entire functions of zero order. Suppose that $q$ is a non-zero complex constant and $n \geq 6$ is an integer. If $f^{n}(z)(f(z)-1) f(q z)$ and $g^{n}(z)(g(z)-1) g(q z)$ share $1 C M$, then $f(z) \equiv g(z)$.

In the year 2011, X. D. Luo, W. C. Lin [22], obtained the following result.

Theorem 6. [22]. Let $f$ and $g$ be transcendental entire functions of finite order, $c$ a non-zero complex constant, let $P(z)$ be a non-zero polynomial and let $n>2 \Gamma_{0}+1$ be an integer, where $\Gamma_{0}=m_{1}+2 m_{2}, m_{1}$ is the number of the simple zero of $P(z)$ and $m_{2}$ is the number of multiple zeros of $P(z)$. If $P(f) f(z+c)$ and $P(g) g(z+c)$ share $1 C M$, then one of the following results holds:

(i) $f=$ tg for a constant $t$ such that $t^{d}=1$, where $d=G C D\left\{\lambda_{0}, \lambda_{1}, \ldots, \lambda_{n}\right\}$ and

$$
\lambda_{i}=\left\{\begin{array}{ll}
i+1, & a_{i} \neq 0 \\
n+1, & a_{i}=0
\end{array} i=0,1,2, \ldots, n ;\right.
$$

(ii) $f$ and $g$ satisfy the algebraic equation $R(f, g) \equiv 0$, where $R\left(\omega_{1}, \omega_{2}\right)=P\left(\omega_{1}\right) \omega_{1}(z+c)-P\left(\omega_{2}\right) \omega_{2}(z+c)$;

(iii) $f(z)=e^{\alpha(z)}, g(z)=e^{\beta(z)}$, where $\alpha(z)$ and $\beta(z)$ are two polynomials, $b$ is a constant satisfying $\alpha+\beta \equiv b$ and $a_{n}^{2} e^{(n+1) b}=1$. 
In 2015, the author's H. Y. Xu, K. Liu and T. B. Cao [25] obtained the following results on finding the uniqueness of $q$-shifts of entire functions.

Theorem 7. [25]. Let $f$ and $g$ be transcendental entire functions of zero order, and let $q \in \mathbb{C} \backslash\{0\}, \eta \in \mathbb{C}, P(f), \Gamma_{0}, d$ be as in Theorem F. If $P(f) f(q z+\eta)$ and $P(g) g(q z+\eta)$ share $1 C M$ and $n>2 \Gamma_{0}+1$, then one of the following cases holds:

(i) $f=\operatorname{tg}$ for a constant $t$ such that $t^{d}=1$;

(ii) $f$ and $g$ satisfy the algebraic equation $R(f, g) \equiv 0$, where $R\left(\omega_{1}, \omega_{2}\right)=P\left(\omega_{1}\right) \omega_{1}(q z+\eta)-P\left(\omega_{2}\right) \omega_{2}(q z+\eta)$;

(iii) $f g=\mu$, where $\mu$ is a complex constant satisfying $a_{n}^{2} \mu^{n+1} \equiv 1$.

Theorem 8. [25]. Under the assumptions of Theorem $G$, if $E_{l}(1 ; P(f) f(q z+\eta))=E_{l}(1 ; P(g) g(q z+\eta))$ and $l, n$, $m$ are integers satisfying one of the following conditions:

(I) $l=2, n>2 \Gamma_{0}+m+2-\lambda$;

(II) $l=1, n>2 \Gamma_{0}+2 m+3-2 \lambda$;

(III) $l=0, n>2 \Gamma_{0}+3 m+4-3 \lambda$;

(IV) $l \geq 3, n>2 \Gamma_{0}+1$.

Then the conclusions of Theorem $G$ hold, where $\lambda=\min \{\Theta(0, f), \Theta(0, g)\}$ and $m$ is stated as above.

The investigation of the author's H. Y. Xu, K. Liu and T. B. Cao motivated the present work. Here our aim is to find the uniqueness of the $q$-shift difference polynomial by considering the function $f^{m}(z) P_{n}(f(q z+\eta)) f^{(k)}(z)$ and $g^{m}(z) P_{n}(g(q z+\eta)) g^{(k)}(z)$, where $P_{n}(f(q z+\eta))$ is the $q$-shift difference polynomial and let $t_{n}$ be the number of distinct zeros of $P_{n}(f(q z+\eta))$.

We now state the main results.

Theorem 9. Let $f$ be a zero-order transcendental meromorphic (resp. entire) functions, $q \in \mathbb{C} \backslash\{0\}, \eta \in \mathbb{C}$. Then, for $n>$ $3 k+t_{n}-m+6\left(n>2 k+t_{n}-m+1\right), f^{m}(z) P_{n}(f(q z+\eta)) f^{(k)}(z)=a(z)$ has infinitely many solutions, where $a(z) \in \mathbb{S}(f) \backslash$ $\{0\}, P_{n}(f(q z+\eta))$ is a non-zero $q$-shift difference polynomial and let $t_{n}$ be the number of distinct zeros of $P_{n}(f(q z+\eta))$.

Theorem 10. Let $f$ and $g$ be transcendental entire functions of zero order, and let $q \in \mathbb{C} \backslash\{0\}, \eta \in \mathbb{C}$, let $P_{n}(f(q z+\eta))$ be a non-zero polynomial and $\Gamma_{0}=m_{1}+2 m_{2}$, where $m_{1}$ is the number of simple zeros and $m_{2}$ is the number of multiple zeros of $P_{n}(f(q z+\eta))$. If $f^{m}(z) P_{n}(f(q z+\eta)) f^{(k)}(z)$ and $g^{m}(z) P_{n}(g(q z+\eta)) g^{(k)}(z)$ share 1 CM and $n>2 k+2 \Gamma_{0}+m+3$, then one of the following cases holds:

(i) $f=t g$ for a constant $t$ such that $t^{d}=1$, where $d=G C D\{m+n+k-1, m+n+k-2, \ldots, m+k-1\}$.

(ii) $f$ and $g$ satisfy the algebraic equation $R(f, g) \equiv 0$, where

$$
\begin{aligned}
R\left(\omega_{1}, \omega_{2}\right)= & \omega_{1}^{m+k-1}\left[\frac{a_{n} \omega_{1}^{n}(q z+\eta)(k-1)}{m+n+k-1}+\frac{a_{n-1} \omega_{1}^{n-1}(q z+\eta)(k-1)}{m+n+k-2}+\ldots+\frac{a_{0}(k-1)}{m+k-1}\right]- \\
& \omega_{2}^{m+k-1}\left[\frac{a_{n} \omega_{2}^{n}(q z+\eta)(k-1)}{m+n+k-1}+\frac{a_{n-1} \omega_{2}^{n-1}(q z+\eta)(k-1)}{m+n+k-2}+\ldots+\frac{a_{0}(k-1)}{m+k-1}\right]
\end{aligned}
$$

(iii) $f g=\mu$, where $\mu$ is a complex constant satisfying $a_{n}^{2} \mu^{n+m+k} \equiv t$.

We require the following definition to state our next theorem.

Definition 1. [15], [16]. Let $l$ be a non-negative integer or infinity. For $a \in \mathbb{C} \cup\{\infty\}$, by $E_{l}(a ; f)$ we denote the set of all a-points of $f$ where an a-point of multiplicity $k$ is counted $k$ times if $k \leq l$ and $l+1$ times if $k>l$. If $E_{l}(a ; f)=E_{l}(a ; g)$, we say that $f, g$ share the value a with weight $l$. 
Theorem 11. Under the assumptions of Theorem 10, if

$$
E_{l}\left(1 ; f^{m}(z) P_{n}(f(q z+\eta)) f^{(k)}(z)\right)=E_{l}\left(1 ; g^{m}(z) P_{n}(g(q z+\eta)) g^{(k)}(z)\right)
$$

and $l, m$ and $t_{n}$ are integers satisfying one of the following conditions:
(I) $l=2, n>\frac{5 k}{2}+2 \Gamma_{0}+\frac{t_{n}}{2}-m-\lambda+\frac{17}{2}$;
(II) $l=1, n>4 k+2 \Gamma_{0}+2 t_{n}-2 \lambda-m+9$;
(III) $l=0, n>5 k+2 \Gamma_{0}+3 t_{n}-3 \lambda-m+13$;
(IV) $l \geq 3, n>2 k+2 \Gamma_{0}-m+7$.

Then the conclusions of Theorem 10. hold, where $\lambda=\min \{\Theta(0, f), \Theta(0, g)\}$ and $t_{n}$ is stated as in Theorem 9.

We also require the following definition and notations in this paper.

For $a \in \mathbb{C} \cup \infty$ and $k$ a positive integer, by $\bar{N}_{(k}\left(r, \frac{1}{f-a}\right)$ we denote the counting function of those $a$-points of $f$ whose multiplicities are not less than $k$ in counting the $a$-points of $f$ we ignore the multiplicities ([11]) and $N_{k}\left(r, \frac{1}{f-a}\right)=\bar{N}\left(r, \frac{1}{f-a}\right)+\bar{N}_{(2}\left(r, \frac{1}{f-a}\right)+\ldots+\bar{N}_{(k}\left(r, \frac{1}{f-a}\right)$.

For $a \in \mathbb{C} \cup \infty$, we define

$$
\Theta(a, f)=1-\limsup _{r \rightarrow \infty} \frac{\bar{N}\left(r, \frac{1}{f-a}\right)}{T(r, f)}
$$

Definition 2. [1]. When $f$ and $g$ share 1 IM, we denote by $\bar{N}_{L}\left(r, \frac{1}{f-1}\right)$ the counting function of those 1-points of $f$ whose multiplicities are greater than 1-points of $g$, where each zero is counted only once; similarly, we have $\bar{N}_{L}\left(r, \frac{1}{g-1}\right)$. Let $z_{0}$ be a zero of $f-1$ of multiplicity $p$ and a zero of $g-1$ of multiplicity $q$, by $N_{11}\left(r, \frac{1}{f-1}\right)$ we also denote the counting function of those 1-points of $f$ and $g$ where $p=q=1$.

\section{Lemmas}

We use the following lemmas for proving our main results.

Lemma 1. [7]. Let $f$ and $g$ be two meromorphic functions. If $f$ and $g$ share $1 C M$, then one of the following three cases holds:

(i) $T(r, f)+T(r, g) \leq 2 N_{2}(r, f)+2 N_{2}(r, g)+2 N_{2}\left(r, \frac{1}{f}\right)+2 N_{2}\left(r, \frac{1}{g}\right)+S(r, f)+S(r, g)$;

(ii) $f \equiv g$;

(iii) $f . g=1$.

Lemma 2. [6]. Let $f$ and $g$ be two meromorphic functions, and let l be a positive integer. If $E_{l}(1 ; f)=E_{l}(1 ; g)$, then one of the following cases must occur:

(i) $\mathrm{T}(\mathrm{r}, \mathrm{f})+\mathrm{T}(\mathrm{r}, \mathrm{g}) \leq N_{2}(r, f)+N_{2}(r, g)+N_{2}\left(r, \frac{1}{f}\right)+N_{2}\left(r, \frac{1}{g}\right)+\bar{N}\left(r, \frac{1}{f-1}\right)$

$$
+\bar{N}\left(r, \frac{1}{g-1}\right)-N_{11}\left(r, \frac{1}{f-1}\right)+\bar{N}_{(l+1}\left(r, \frac{1}{f-1}\right)+\bar{N}_{(l+1}\left(r, \frac{1}{g-1}\right)+S(r, f)+S(r, g) .
$$

(ii) $f=\frac{(b+1) g+(a-b-1)}{b g+(a-b)}$, where $a(\neq 0), b$ are two constants. 
Lemma 3. [6]. Let $f$ and $g$ be two meromorphic functions. If $f$ and $g$ share 1 IM, then one of the following cases must occur:

$$
\text { (i) } \begin{aligned}
\mathrm{T}(\mathrm{r}, \mathrm{f})+\mathrm{T}(\mathrm{r}, \mathrm{g}) & \leq 2\left[N_{2}(r, f)+N_{2}\left(r, \frac{1}{f}\right)+N_{2}(r, g)+N_{2}\left(r, \frac{1}{g}\right)\right] \\
& +3 \bar{N}_{L}\left(r, \frac{1}{f-1}\right)+3 \bar{N}_{L}\left(r, \frac{1}{g-1}\right)+S(r, f)+S(r, g) ;
\end{aligned}
$$

(ii) $f=\frac{(b+1) g+(a-b-1)}{b g+(a-b)}$, where $a(\neq 0), b$ are two constants.

Lemma 4. [28]. Let $f$ be a non-constant meromorphic function and $P(f)=a_{0}+a_{1} f+a_{2} f^{2}+\ldots+a_{n} f^{n}$, where $a_{0}, a_{1}, a_{2}, . ., a_{n}$ are constants and $a_{n} \neq 0$. Then

$$
T(r, P(f))=n T(r, f)+S(r, f) .
$$

Lemma 5. [9]. Let $f$ be a non-constant meromorphic function, $s>0, \alpha<1$, and let $F \subset \mathbb{R}_{+}$be the set of all $r$ such that $T(r, f) \leq \alpha T(r+s, f)$. If the logarithmic measure of $F$ is infinite, that is, $\int_{F} \frac{d t}{t}=\infty$, then $f$ is of infinite order of growth.

Lemma 6. [5]. Let $f(z)$ be a meromorphic function of finite order $\rho$ and $c$ a non-zero complex constant. Then, for each $\varepsilon>0$, we have

$$
T(r, f(z+c))=T(r, f(z))+O\left(r^{\rho-1+\varepsilon}\right)+O(\log r) .
$$

Lemma 7. [30]. Let $f(z)$ be a transcendental meromorphic function of zero order and $q$ a non-zero complex constant. Then

$$
T(r, f(q z))=(1+o(1)) T(r, f(z))
$$

and

$$
N(r, f(q z))=(1+o(1)) N(r, f(z))
$$

on a set of logarithmic density 1.

Remark. [25]. Under the assumptions of Lemma 2.7, from the definition of $S_{1}(r, f)$ we have

$$
\begin{aligned}
& T(r, f(q z))=T(r, f(z))+S_{1}(r, f), \\
& N(r, f(q z))=N(r, f(z))+S_{1}(r, f) .
\end{aligned}
$$

Lemma 8. [25]. Let $f(z)$ be a transcendental meromorphic function of zero order and q, $\eta$ two non-zero complex constants. Then

$$
\begin{aligned}
T(r, f(q z+\eta)) & =T(r, f(z))+S_{1}(r, f), \\
N\left(r, \frac{1}{f(q z+\eta)}\right) & \leq N\left(r, \frac{1}{f}\right)+S_{1}(r, f), \\
N(r, f(q z+\eta)) & \leq N(r, f)+S_{1}(r, f), \\
\bar{N}\left(r, \frac{1}{f(q z+\eta)}\right) & \leq \bar{N}\left(r, \frac{1}{f}\right)+S_{1}(r, f), \\
\bar{N}(r, f(q z+\eta)) & \leq \bar{N}(r, f)+S_{1}(r, f) .
\end{aligned}
$$

Lemma 9. [20]. Let $f(z)$ be a non-constant zero order meromorphic function and $q \in \mathbb{C} \backslash\{0\}$. Then

$$
m\left(r, \frac{f(q z+\eta)}{f(z)}\right)=S(r, f)
$$


on a set of logarithmic density 1.

Lemma 10. [25], Lemma 12. Let $f(z)$ and $g(z)$ be transcendental entire functions of zero order. $P(z)$ be a non-zero polynomial as stated above. If $n \geq 2$, and

$$
f(z)^{m} P_{n}(f(q z+\eta)) f^{(k)}(z) g(z)^{m} P_{n}(g(q z+\eta)) g^{(k)}(z) \equiv t
$$

where $q(\neq 0), \eta, t(\neq 0)$ are complex constants, then we have $f g=\mu$, where $a_{n}^{2} \mu^{n+m+k}=t$.

Proof. Proof of the lemma here is similar to the proof of Lemma 12 in [25].

Lemma 11. Let $f$ be a transcendental meromorphic function of zero order, $q(\neq 0), \eta$ be complex constants, and let $P_{n}(f(q z+\eta))=a_{n} f^{n}(q z+\eta)+a_{n-1} f^{n-1}(q z+\eta)+\ldots+a_{1} f(q z+\eta)+a_{0}$. Also, $F(z)=f^{m}(z) P_{n}(f(q z+\eta)) f^{(k)}(z)$. Then, we have

$$
(m+n-1-k) T(r, f)+S_{1}(r, f) \leq T(r, F(z)) \leq(m+n+1+k) T(r, f)+S_{1}(r, f) .
$$

If $f$ is a transcendental entire function of zero order, we have

$$
T(r, F(z))=T\left(r, f^{m}(z) P_{n}(f(q z+\eta)) f^{(k)}(z)\right)=(m+n+1) T(r, f)+S_{1}(r, f)
$$

Proof. Let $f$ be a transcendental entire function of zero order. Also, let $F(z)=f^{m}(z) P_{n}(f(q z+\eta)) f^{(k)}(z)$. We have, $T\left(r, P_{n}(f(q z+\eta))\right)=n T(r, f(q z+\eta))=n T(r, f)+S_{1}(r, f)$.

Consider,

$$
\begin{aligned}
& T(r, F(z))=m(r, F(z)) \\
& \leq m\left(r, f^{m}(z) P_{n}(f(q z+\eta)) f(z)\right)+m\left(r, \frac{f^{(k)}(z)}{f(z)}\right) \\
& \leq T\left(r, f^{m}(z) P_{n}(f(q z+\eta)) f(z)\right)+S_{1}(r, f) \\
& \text { i.e., } T(r, F(z)) \leq(m+n+1) T(r, f)+S_{1}(r, f)
\end{aligned}
$$

On the otherhand, let us consider

$$
\begin{aligned}
& \text { i.e., }(m+n+1) T(r, f)=T\left(r, f^{m}(z) P_{n}(f(q z+\eta)) f(z)\right)+S(r, f) \\
& \leq m\left(r, f^{m}(z) P_{n}(f(q z+\eta)) f^{(k)}(z)\right)+m\left(r, \frac{f(z)}{f^{(k)}(z)}\right)+S(r, f) \\
& \leq T(r, F(z))+S(r, f) \\
& \text { i.e., }(m+n+1) T(r, f)+S_{1}(r, f) \leq T(r, F(z))
\end{aligned}
$$

By (1), (2), we have

$$
T(r, F(z))=T\left(r, f^{m}(z) P_{n}(f(q z+\eta)) f^{(k)}(z)\right)=(m+n+1) T(r, f)+S_{1}(r, f) .
$$


If $f$ is a transcendental meromorphic function of zero order, from Lemma 8 and Lemma 4, we have

$$
\begin{aligned}
T\left(r, f^{m}(z) P_{n}(f(q z+\eta)) f^{(k)}(z)\right) & \leq T\left(r, f^{m}(z)\right)+T\left(r, P_{n}(f(q z+\eta))\right)+T\left(r, f^{(k)}(z)\right)+S_{1}(r, f) . \\
& \leq(m+n+1+k) T(r, f)+S_{1}(r, f) .
\end{aligned}
$$

On the otherhand, by Lemma 4, we have

$$
\begin{aligned}
(m+n+1) T(r, f) & =T\left(r, f^{m}(z) P_{n}(f(q z+\eta)) f(z)\right)+S(r, f) \\
& \leq T(r, F(z))+T\left(r, \frac{f(z)}{f^{(k)}(z)}\right)+S(r, f) \\
& \leq T(r, F(z))+(2+k) T(r, f)+S(r, f) \\
\text { i.e., } T(r, F(z)) & \geq(m+n-1-k) T(r, f)+S_{1}(r, f) .
\end{aligned}
$$

By (3), (4), we get

$$
(m+n-1-k) T(r, f)+S_{1}(r, f) \leq T(r, F(z)) \leq(m+n+1+k) T(r, f)+S_{1}(r, f) .
$$

\section{Proof of Theorems}

Proof. (Proof of Theorem 9)

Case 1. Let $f$ be a transcendental meromorphic function of zero order.

Let us suppose that $f^{m}(z) P_{n}(f(q z+\eta)) f^{(k)}(z)=\alpha(z)$ has finitely many solutions. From Lemma 11, we have $S\left(r, f^{m}(z) P_{n}(f(q z+\eta)) f^{(k)}(z)\right)=S(r, f)$. By using the second fundamental theorem, Lemma 8 and the definition of $t_{n}$, we have

$$
\begin{aligned}
T\left(r, f^{m}(z) P_{n}(f(q z+\eta)) f^{(k)}(z)\right) & \leq \bar{N}\left(r, f^{m}(z) P_{n}(f(q z+\eta)) f^{(k)}(z)\right)+\bar{N}\left(r, \frac{1}{f^{m}(z) P_{n}(f(q z+\eta)) f^{(k)}(z)}\right) \\
& +\bar{N}\left(r, \frac{1}{f^{m}(z) P_{n}(f(q z+\eta)) f^{(k)}(z)-\alpha(z)}\right)+S(r, f) \\
& \leq 3 \bar{N}(r, f)+\bar{N}\left(r, \frac{1}{f}\right)+t_{n} \bar{N}\left(r, \frac{1}{f}\right)+N_{1+k}\left(r, \frac{1}{f}\right)+k \bar{N}(r, f)+S(r, f) \\
& \leq\left(2 k+t_{n}+5\right) T(r, f)+S(r, f)
\end{aligned}
$$

i.e., $(m+n-1-k) T(r, f)+S(r, f) \leq\left(2 k+t_{n}+5\right) T(r, f)+S(r, f)$, i.e., $n \leq 3 k+t_{n}-m+6$, which is a contradiction to $n>3 k+t_{n}-m+6$.

Case 2. Let $f$ be a transcendental entire function of zero order. Let us suppose that $f^{m}(z) P_{n}(f(q z+\eta)) f^{(k)}(z)=\alpha(z)$ has finitely many solutions. By using the same argument as in Case 1 and (4), we get

$$
\begin{aligned}
T\left(r, f^{m}(z) P_{n}(f(q z+\eta)) f^{(k)}(z)\right) & \leq \bar{N}\left(r, \frac{1}{f^{m}(z) P_{n}(f(q z+\eta)) f^{(k)}(z)}\right)+S(r, f) \\
& \leq\left(2 k+t_{n}+2\right) T(r, f)+S(r, f)
\end{aligned}
$$


i.e., $(m+n+1) T(r, f)+S(r, f) \leq\left(2 k+t_{n}+2\right) T(r, f)+S(r, f)$, i.e., $n \leq 2 k+t_{n}-m+1$, which is a contradiction to $n>2 k+t_{n}-m+1$. This completes proof of Theorem 9 .

Proof. (Proof of Theorem 10)

Let $F(z)=f^{m}(z) P_{n}(f(q z+\eta)) f^{(k)}(z), G(z)=g^{m}(z) P_{n}(g(q z+\eta)) g^{(k)}(z)$. By the assumption of Theorem ??, we have $F(z), G(z)$ share $1 \mathrm{CM}$. Then, the following three cases will arise in view of Lemma 1.

Case 1. Suppose that $F(z), G(z)$ satisfy Lemma 1 (i), we have

$$
T(r, F(z))+T(r, G(z)) \leq 2 N_{2}(r, F)+2 N_{2}(r, G)+2 N_{2}\left(r, \frac{1}{F}\right)+2 N_{2}\left(r, \frac{1}{G}\right)+S(r, F)+S(r, G) .
$$

Since $f(z), g(z)$ are entire functions of zero order, by using Lemma 8, Lemma 11, we have $S(r, F)=S(r, f), S(r, G)=$ $S(r, g)$.

$$
\begin{aligned}
T(r, F(z))+T(r, G(z)) \leq & 2 N_{2}\left(r, \frac{1}{F}\right)+2 N_{2}\left(r, \frac{1}{G}\right)+S(r, f)+S(r, g) \\
& \leq 2 N_{2}\left(r, \frac{1}{f(z)^{m}}\right)+2 N_{2}\left(r, \frac{1}{P_{n}(f(q z+\eta))}\right) \\
& +2 N_{2}\left(r, \frac{1}{f^{(k)}(z)}\right)+2 N_{2}\left(r, \frac{1}{g(z)^{m}}\right)+2 N_{2}\left(r, \frac{1}{P_{n}(g(q z+\eta))}\right) \\
& +2 N_{2}\left(r, \frac{1}{g^{(k)}(z)}\right)+S(r, f)+S(r, g) \\
& \leq\left(2 m+2 \Gamma_{0}+2 k+4\right)(T(r, f)+T(r, g))+S(r, f)+S(r, g) \\
\text { i.e., }(m+n+1)(T(r, f)+T(r, g)) \leq & \left(2 m+2 \Gamma_{0}+2 k+4\right)(T(r, f)+T(r, g))+S(r, f)+S(r, g)
\end{aligned}
$$

On simplifying, we get $n \leq 2 k+2 \Gamma_{0}+m+3$, which contradicts $n>2 k+2 \Gamma_{0}+m+3$.

Case 2. If $F(z) \equiv G(z)$, that is,

$$
f(z)^{m} P_{n}(f(q z+\eta)) f^{(k)}(z)=g(z)^{m} P_{n}(g(q z+\eta)) g^{(k)}(z) .
$$

Let $h(z)=\frac{f(z)}{g(z)}$.

Subcase 1.1. Suppose that $h(z)$ is a constant. On Integrating (5), we get

$$
\begin{aligned}
& f^{m+k-1}\left[\frac{a_{n} f^{n}(q z+\eta)(k-1)}{m+n+k-1}+\frac{a_{n-1} f^{n-1}(q z+\eta)(k-1)}{m+n+k-2}+\ldots+\frac{a_{0}(k-1)}{m+k-1}\right] \\
& =g^{m+k-1}\left[\frac{a_{n} g^{n}(q z+\eta)(k-1)}{m+n+k-1}+\frac{a_{n-1} g^{n-1}(q z+\eta)(k-1)}{m+n+k-2}+\ldots+\frac{a_{0}(k-1)}{m+k-1}\right]
\end{aligned}
$$

On substituting $f=g h$ in (6), we get

$g^{m+k-1}\left[\frac{a_{n} g^{n}(q z+\eta)(k-1)}{m+n+k-1}\left(h^{m+n+k-1}-1\right)+\frac{a_{n-1} g^{n-1}(q z+\eta)(k-1)}{m+n+k-2}\left(h^{m+n+k-2}-1\right)+\ldots+\frac{a_{0}(k-1)}{m+k-1}\left(h^{m+k-1}-1\right)\right] \equiv 0$

Since $g$ is a transcendental entire function, $g^{m+k-1} \neq 0$. Hence,

$$
\frac{a_{n} g^{n}(q z+\eta)(k-1)}{m+n+k-1}\left(h^{m+n+k-1}-1\right)+\frac{a_{n-1} g^{n-1}(q z+\eta)(k-1)}{m+n+k-2}\left(h^{m+n+k-2}-1\right)+\ldots+\frac{a_{0}(k-1)}{m+k-1}\left(h^{m+k-1}-1\right) \equiv 0 .
$$


By (7), we get $h^{d}=1$, where $d=G C D\{m+n+k-1, m+n+k-2, \ldots, m+k-1\}, a_{n-i} \neq 0$, for some $i=0,1,2, \ldots, n$. Thus, $f=t g$, where $t^{d}=1$.

Subcase 1.2. Suppose that $h(z)$ is not a constant. Then, by (6), $f$ and $g$ satisfy the algebraic equation $R(f, g) \equiv 0$, where

$$
\begin{aligned}
R\left(\omega_{1}, \omega_{2}\right)= & \omega_{1}^{m+k-1}\left[\frac{a_{n} \omega_{1}^{n}(q z+\eta)(k-1)}{m+n+k-1}+\frac{a_{n-1} \omega_{1}^{n-1}(q z+\eta)(k-1)}{m+n+k-2}+\ldots+\frac{a_{0}(k-1)}{m+k-1}\right]- \\
& \omega_{2}^{m+k-1}\left[\frac{a_{n} \omega_{2}^{n}(q z+\eta)(k-1)}{m+n+k-1}+\frac{a_{n-1} \omega_{2}^{n-1}(q z+\eta)(k-1)}{m+n+k-2}+\ldots+\frac{a_{0}(k-1)}{m+k-1}\right] .
\end{aligned}
$$

Subcase 1.3. If $F(z) G(z) \equiv 1$. By using Lemma 2.10, we get $f g=\mu$, for a constant $\mu$ such that $a_{n}^{2} \mu^{n+m+k}=t$. This completes proof of Theorem 1.2.

Proof. (Proof of Theorem 11)

From the assumptions of Theorem 1.3., we have $E_{l}(1 ; F(z))=E_{l}(1 ; G(z))$. Then,

(I) $l=2$. Since

$$
\begin{aligned}
\bar{N}\left(r, \frac{1}{F-1}\right)+\bar{N}\left(r, \frac{1}{G-1}\right) & -N_{11}\left(r, \frac{1}{F-1}\right)+\frac{1}{2} \bar{N}_{(l+1}\left(r, \frac{1}{F-1}\right)+\frac{1}{2} \bar{N}_{(l+1}\left(r, \frac{1}{G-1}\right) . \\
& \leq \frac{1}{2} N\left(r, \frac{1}{F-1}\right)+\frac{1}{2} N\left(r, \frac{1}{G-1}\right) \\
& \leq \frac{1}{2} T(r, F)+\frac{1}{2} T(r, G)+S(r, F)+S(r, G)
\end{aligned}
$$

and

$$
\begin{aligned}
\bar{N}_{(l+1}\left(r, \frac{1}{F-1}\right) & \leq \frac{1}{2} N\left(r, \frac{F}{F^{\prime}}\right)=\frac{1}{2} N\left(r, \frac{F^{\prime}}{F}\right)+S(r, F) \\
& \leq \frac{1}{2} \bar{N}\left(r, \frac{1}{F}\right)+S(r, F) \\
& \leq\left(\frac{k+t_{n}+1}{2}\right) T(r, f)+\frac{1}{2} \bar{N}\left(r, \frac{1}{f}\right)+S(r, f) .
\end{aligned}
$$

Similarly,

$$
\bar{N}_{(l+1}\left(r, \frac{1}{G-1}\right) \leq\left(\frac{k+t_{n}+1}{2}\right) T(r, g)+\frac{1}{2} \bar{N}\left(r, \frac{1}{g}\right)+S(r, g) .
$$

Case 1. Let $F(z), G(z)$ satisfy Lemma 1 (i). Since $f(z), g(z)$ are transcendental entire functions and (8), we have

$$
\begin{aligned}
T(r, F(z))+T(r, G(z)) & \leq 2 N_{2}\left(r, \frac{1}{F}\right)+2 N_{2}\left(r, \frac{1}{G}\right)+\bar{N}_{(l+1}\left(r, \frac{1}{F-1}\right) \\
& +\bar{N}_{(l+1}\left(r, \frac{1}{G-1}\right)+S(r, f)+S(r, g) .
\end{aligned}
$$

By using Lemma 11 and let $\lambda=\min \{\Theta(0, f), \Theta(0, g)\}$ and on simplifying, we get

$$
\left[n-\left(\frac{4 \Gamma_{0}+5 k+t_{n}+17}{2}\right)+m+\lambda\right](T(r, f)+T(r, g)) \leq S(r, f)+S(r, g)
$$


That is, $n \leq \frac{5 k}{2}+2 \Gamma_{0}+\frac{t_{n}}{2}-m-\lambda+\frac{17}{2}$, which contradicts

$$
n>\frac{5 k}{2}+2 \Gamma_{0}+\frac{t_{n}}{2}-m-\lambda+\frac{17}{2}
$$

Case 2. Let $F(z), G(z)$ satisfy Lemma 2 (ii), that is,

$$
F=\frac{(b+1) G+(a-b-1)}{b G+(a-b)},
$$

where $a(\neq 0), b$ are two constants. We now consider the following three cases.

Subcase 2.1. $b \neq 0,-1$. If $a-b-1 \neq 0$, then by (10), we have

$$
\bar{N}\left(r, \frac{1}{G+\frac{a-b-1}{b+1}}\right)=\bar{N}\left(r, \frac{1}{F}\right) .
$$

Since $f, g$ are entire functions of zero order, by using the Second Fundamental Theorem and Lemma 7 and Lemma 8 , we have

$$
\begin{aligned}
T(r, G) & \leq \bar{N}\left(r, \frac{1}{G}\right)+\bar{N}\left(r, \frac{1}{G+\frac{a-b-1}{b+1}}\right)+S(r, g) \\
& \leq \bar{N}\left(r, \frac{1}{G}\right)+\bar{N}\left(r, \frac{1}{F}\right)+S(r, g)
\end{aligned}
$$

By using Lemma 11 and simplifying, we get

$$
(m+n+1) T(r, g)+S(r, g) \leq\left(k+t_{n}+2\right)(T(r, f)+T(r, g))+S(r, f)+S(r, g)
$$

Similarly,

$$
(m+n+1) T(r, f)+S(r, f) \leq\left(k+t_{n}+2\right)(T(r, f)+T(r, g))+S(r, f)+S(r, g)
$$

On adding, we get

$$
(m+n+1)(T(r, f)+T(r, g)) \leq\left(2 k+2 t_{n}+4\right)(T(r, f)+T(r, g))+S(r, f)+S(r, g)
$$

That is, $n \leq 2 k+2 t_{n}-m+3$, which contradicts (9). If $a-b-1=0$, then by (10), we know

$$
F=\frac{(b+1) G}{b G+1}
$$

Since $f, g$ are entire functions, we get that $\frac{-1}{b}$ is a Picard's exceptional value of $G(z)$. By the second main theorem, we get

$$
T(r, G) \leq \bar{N}\left(r, \frac{1}{G}\right)+S(r, G) .
$$

By Lemma 11, we have

$$
(m+n+1) T(r, g)+S(r, g) \leq\left(k+t_{n}+2\right) T(r, g)+S(r, g)
$$


That is, $n \leq k+t_{n}-m+1$, which contradicts (9).

Subcase 2.2. $b=-1$. Then, (10) becomes,

$$
F=\frac{a}{a+1-G} .
$$

If $a+1 \neq 0$, then $a+1$ is a Picard's exceptional value of $G$. Similarly, to the above discussion in Subcase 2.1., we deduce a contradiction.

If $a+1=0$, then $F G=1$, that is

$$
f(z)^{m} P_{n}(f(q z+\eta)) f^{(k)}(z) g(z)^{m} P_{n}(g(q z+\eta)) g^{(k)}(z) \equiv 1
$$

Since $n>\frac{5 k}{2}+2 \Gamma_{0}+\frac{t_{n}}{2}-m-\lambda+\frac{17}{2} \geq 9$, by Lemma 2.10, we get $f g=\mu$, for a constant $\mu$ such that $a_{n}^{2} \mu^{n+m+k} \equiv t$.

Subcase 2.3. $b=0$. Then, (10) becomes,

$$
F=\frac{G+a-1}{a}
$$

If $a-1 \neq 0$, then

$$
\bar{N}\left(r, \frac{1}{G+a-1}\right)=\bar{N}\left(r, \frac{1}{F}\right) .
$$

Similarly, to the discussion in Subcase 2.1, we get a contradiction. If $a-1=0$, then $F \equiv G$, that is

$$
f(z)^{m} P_{n}(f(q z+\eta)) f^{(k)}(z)=g(z)^{m} P_{n}(g(q z+\eta)) g^{(k)}(z)
$$

By using the same argument as in the proof of Case 2 of Theorem ??, we get the same conclusion.

(II) $l=1$. Since

$$
\begin{aligned}
\bar{N}\left(r, \frac{1}{F-1}\right)+\bar{N}\left(r, \frac{1}{G-1}\right)-N_{11}\left(r, \frac{1}{F-1}\right) & \leq \frac{1}{2} N\left(r, \frac{1}{F-1}\right)+\frac{1}{2} N\left(r, \frac{1}{G-1}\right) \\
& \leq \frac{1}{2} T(r, F)+\frac{1}{2} T(r, G)+S(r, F)+S(r, G)
\end{aligned}
$$

From Lemma 8, we have

$$
\begin{aligned}
\bar{N}_{(2}\left(r, \frac{1}{F-1}\right) & \leq N\left(r, \frac{F}{F^{\prime}}\right)=N\left(r, \frac{F^{\prime}}{F}\right)+S(r, f) \\
& \leq \bar{N}\left(r, \frac{1}{F}\right)+S(r, f) \\
& \leq\left(k+1+t_{n}\right) T(r, f)+\bar{N}\left(r, \frac{1}{f}\right)+S(r, f),
\end{aligned}
$$

and

$$
\bar{N}_{(2}\left(r, \frac{1}{G-1}\right) \leq\left(k+1+t_{n}\right) T(r, g)+\bar{N}\left(r, \frac{1}{g}\right)+S(r, g)
$$


Case 1. If $F(z), G(z)$ satisfy Lemma 2 (i), since $f, g$ are entire functions and by (11), (12), we get

$$
\begin{aligned}
T(r, F)+T(r, G) & \leq N_{2}\left(r, \frac{1}{F}\right)+N_{2}\left(r, \frac{1}{G}\right)+\bar{N}\left(r, \frac{1}{F-1}\right) \\
& +\bar{N}\left(r, \frac{1}{G-1}\right)-N_{11}\left(r, \frac{1}{F-1}\right)+\bar{N}_{(2}\left(r, \frac{1}{F-1}\right) \\
& +\bar{N}_{(2}\left(r, \frac{1}{G-1}\right)+S(r, F)+S(r, G) .
\end{aligned}
$$

By using Lemma 11 and simplifying, we get $n \leq 4 k+2 \Gamma_{0}+2 t_{n}-2 \lambda-m+11$, which contradicts $n>4 k+2 \Gamma_{0}+2 t_{n}-2 \lambda-m+11$.

Case 2. If $F(z), G(z)$ satisfy Lemma 2 (ii). Similar to the proof of Case 2 in (I), we get the conclusions of Theorem 11.

(III) $l=0$. Then $F(z), G(z)$ share 1 IM. From the definitions of $F(z), G(z)$, we have

$$
\begin{aligned}
\bar{N}_{L}\left(r, \frac{1}{F-1}\right) \leq N\left(r, \frac{F}{F^{\prime}}\right) & =N\left(r, \frac{F^{\prime}}{F}\right)+S(r, F) \leq \bar{N}\left(r, \frac{1}{F}\right)+S(r, F) \\
& \leq\left(k+t_{n}+1\right) T(r, f)+\bar{N}\left(r, \frac{1}{f}\right)+S(r, f)
\end{aligned}
$$

Similarly, we have

$$
\bar{N}_{L}\left(r, \frac{1}{G-1}\right) \leq\left(k+t_{n}+1\right) T(r, g)+\bar{N}\left(r, \frac{1}{g}\right)+S(r, g)
$$

Case 1. Let $F(z), G(z)$ satisfy Lemma 3 (i). From (13), (14), we get

$$
\begin{aligned}
T(r, F(z))+T(r, G(z)) & \leq 2\left[N_{2}\left(r, \frac{1}{F}\right)+N_{2}\left(r, \frac{1}{G}\right)\right]+3 \bar{N}_{L}\left(r, \frac{1}{F-1}\right) \\
& +3 \bar{N}_{L}\left(r, \frac{1}{G-1}\right)+S(r, F)+S(r, G) .
\end{aligned}
$$

By using Lemma 11 and simplifying, we get $n \leq 5 k+2 \Gamma_{0}+3 t_{n}-m-3 \lambda+13$, which contradicts $n>5 k+2 \Gamma_{0}+3 t_{n}-m-3 \lambda+13$.

Case 2. If $F(z), G(z)$ satisfy Lemma 3 (ii). Similar to the proof of Case 2 in (I), we get the conclusions of Theorem 11.

(IV) $l \geq 3$. Since

$$
\begin{aligned}
\bar{N}\left(r, \frac{1}{F(z)-1}\right) & +\bar{N}\left(r, \frac{1}{G(z)-1}\right)+\bar{N}_{(l+1}\left(r, \frac{1}{F(z)-1}\right) \\
& +\bar{N}_{(l+1}\left(r, \frac{1}{G(z)-1}\right)-N_{11}\left(r, \frac{1}{F(z)-1}\right) \\
& \leq \frac{1}{2} N\left(r, \frac{1}{F(z)-1}\right)+\frac{1}{2} N\left(r, \frac{1}{G(z)-1}\right)+S(r, F)+S(r, G) \\
& \leq \frac{1}{2} T(r, F)+\frac{1}{2} T(r, G)+S(r, F)+S(r, G) .
\end{aligned}
$$


Case 1. Let $F(z), G(z)$ satisfy Lemma 2 (i). By using Lemma 8, Lemma 9 and Lemma 11, we get

$$
\left(n-2 k-2 \Gamma_{0}+m-7\right)(T(r, f)+T(r, g)) \leq S(r, f)+S(r, g) .
$$

That is, $n \leq 2 k+2 \Gamma_{0}-m+7$, which contradicts $n>2 k+2 \Gamma_{0}-m+7$.

Case 2. Let $F(z), G(z)$ satisfy Lemma 2 (ii). Similar to the proof of Case 2 in (I), we get the conclusions of Theorem 11. This completes the proof of Theorem 11.

\section{Conclusion}

By considering the $q$-shift difference polynomial in the functions of the form $f^{m}(z) P_{n}(f(q z+\eta)) f^{(k)}(z)$ and $g^{m}(z) P_{n}(g(q z+\eta)) g^{(k)}(z)$, along with weighted sharing concept in Theorem 11, we prove important analogous results for transcendental meromorphic (resp. entire) functions of zero order.

\section{Competing interests}

The authors declare that they have no competing interests.

\section{Authors' contributions}

All authors have contributed to all parts of the article. All authors read and approved the final manuscript.

\section{References}

[1] Banerjee, Abhijit. Weighted sharing of a small function by a meromorphic function and its derivative. Comput. Math. Appl. 53 (2007), no. 11, 1750-1761.

[2] Barnett, D. C.; Halburd, R. G.; Morgan, W.; Korhonen, R. J. Nevanlinna theory for the $q$-difference operator and meromorphic solutions of $q$-difference equations. Proc. Roy. Soc. Edinburgh Sect. A 137 (2007), no. 3, 457-474.

[3] Charak, K. S.; Korhonen, R. J.; Kumar, Gaurav. A note on partial sharing of values of meromorphic functions with their shifts. J. Math. Anal. Appl. 435 (2016), no. 2, 1241-1248.

[4] Chen, Zongxuan; Huang, Zhibo; Zheng, Xiumin. On properties of difference polynomials. Acta Math. Sci. Ser. B (Engl. Ed.) 31 (2011), no. 2, 627-633.

[5] Chiang, Yik-Man; Feng, Shao-Ji. On the Nevanlinna characteristic of $f(z+\eta)$ and difference equations in the complex plane. Ramanujan J. 16 (2008), no. 1, 105-129.

[6] Fang, Cai-Yun; Fang, Ming-Liang. Uniqueness of meromorphic functions and differential polynomials. Comput. Math. Appl. 44 (2002), no. 5-6, 607-617.

[7] Fang, Mingliang; Hua, Xinhou. Entire functions that share one value. Nanjing Daxue Xuebao Shuxue Bannian Kan 13 (1996), no. $1,44-48$.

[8] Halburd, R. G.; Korhonen, R. J. Difference analogue of the lemma on the logarithmic derivative with applications to difference equations. J. Math. Anal. Appl. 314 (2006), no. 2, 477-487.

[9] Halburd, R. G.; Korhonen, R. J. Finite-order meromorphic solutions and the discrete Painlevï $i \frac{1}{2}$ equations. Proc. Lond. Math. Soc. (3) 94 (2007), no. 2, 443-474.

[10] Halburd, R. G.; Korhonen, R. J. Nevanlinna theory for the difference operator. Ann. Acad. Sci. Fenn. Math. 31 (2006), no. 2 , $463-478$. 
[11] Hayman, W. K. Meromorphic functions. Oxford Mathematical Monographs Clarendon Press, Oxford 1964 xiv+191 pp.

[12] Heittokangas, J.; Korhonen, R.; Laine, I.; Rieppo, J. Uniqueness of meromorphic functions sharing values with their shifts. Complex Var. Elliptic Equ. 56 (2011), no. 1-4, 81-92.

[13] Heittokangas, J.; Korhonen, R.; Laine, I.; Rieppo, J.; Zhang, J. Value sharing results for shifts of meromorphic functions, and sufficient conditions for periodicity. J. Math. Anal. Appl. 355 (2009), no. 1, 352-363.

[14] Huang, Zhi-Bo. Value distribution and uniqueness on $q$-differences of meromorphic functions. Bull. Korean Math. Soc. 50 (2013), no. $4,1157-1171$.

[15] Lahiri, Indrajit. Weighted sharing and uniqueness of meromorphic functions. Nagoya Math. J. 161 (2001), $193-206$.

[16] Lahiri, Indrajit. Weighted value sharing and uniqueness of meromorphic functions. Complex Variables Theory Appl. 46 (2001), no. 3, 241-253.

[17] Laine, Ilpo; Yang, Chung-Chun. Value distribution of difference polynomials. Proc. Japan Acad. Ser. A Math. Sci. 83 (2007), no. $8,148-151$.

[18] Liu, Kai. Meromorphic functions sharing a set with applications to difference equations. J. Math. Anal. Appl. 359 (2009), no. 1, 384-393.

[19] Liu, Kai; Liu, Xinling; Cao, TingBin. Value distributions and uniqueness of difference polynomials. Adv. Difference Equ. 2011, Art. ID 234215, 12 pp.

[20] Liu, Kai; Qi, Xiao-Guang. Meromorphic solutions of $q$-shift difference equations. Ann. Polon. Math. 101 (2011), no. 3, $215-225$.

[21] Liu, Kai; Yang, Lian-Zhong. Value distribution of the difference operator. Arch. Math. (Basel) 92 (2009), no. 3, $270-278$.

[22] Luo, Xudan; Lin, Wei-Chuan. Value sharing results for shifts of meromorphic functions. J. Math. Anal. Appl. 377 (2011), no. 2, $441-449$.

[23] Wang, Xin-Li; Xu, Hong-Yan; Zhan, Tang-Sen. Properties of $q$-shift difference-differential polynomials of meromorphic functions. Adv. Difference Equ. 2014, 2014:249, 16 pp.

[24] Xu, Hong-Yan. On the value distribution and uniqueness of difference polynomials of meromorphic functions. Adv. Difference Equ. 2013, 2013:90, 15 pp.

[25] Xu, Hong Yan; Liu, Kai; Cao, Ting Bin. Uniqueness and value distribution for $q$-shifts of meromorphic functions. Math. Commun. 20 (2015), no. 1, 97-112.

[26] Yang, Lo. Value distribution theory. Translated and revised from the 1982 Chinese original. Springer-Verlag, Berlin; Science Press Beijing, Beijing, 1993. xii+269 pp. ISBN: 3-540-54379-1

[27] Yang, Chung-Chun; Hua, Xinhou. Uniqueness and value-sharing of meromorphic functions. Ann. Acad. Sci. Fenn. Math. 22 (1997), no. 2, 395-406.

[28] Yang, Chung-Chun; Yi, Hong-Xun. Uniqueness theory of meromorphic functions. Mathematics and its Applications, 557. Kluwer Academic Publishers Group, Dordrecht, 2003. viii+569 pp. ISBN: 1-4020-1448-1

[29] Zhang, Jilong. Value distribution and shared sets of differences of meromorphic functions. J. Math. Anal. Appl. 367 (2010), no. 2, 401-408.

[30] Zhang, Jilong; Korhonen, Risto. On the Nevanlinna characteristic of $f(q z)$ and its applications. J. Math. Anal. Appl. 369 (2010), no. $2,537-544$. 